\title{
On some properties of socle-regular Abelian $p$-groups
}

\author{
Peter V. DANCHeV
}

\section{ABSTRACT.}

We establish some crucial properties of socle-regular groups of special type C, thus extending the fully transitive groups of type A introduced by Carroll-Goldsmith in Proc. Royal Irish Acad. - Math. (1996). We also obtain certain other critical properties of subclasses of socle-regular groups of special types D and E.

\section{Department of MATHEMATics}

PlovdiV UNIVERSITY "P. HILENDARSKI"

PLOVDIV 4000, BULGARIA

E-mail address: pvdanchev@yahoo.com

E-mail address: peter.danchev@yahoo.com 